\title{
Should we recommend PEC and when?
}

Author

Institution
Emmanuel Coron

Centre Hospitalier Universitaire, Gastroentérologie et Hépatologie, Nantes, France submitted 20. March 2016 accepted after revision 23. March 2016

\section{Bibliography}

Dol http://dx.doi.org/

10.1055/s-0042-105513

Published online: 29.6.2016

Endoscopy International Open 2016; 04: E742-E743

(c) Georg Thieme Verlag KG Stuttgart · New York

E-ISSN 2196-9736

\section{Corresponding author} Emmanuel Coron

Centre Hospitalier Universitaire Gastroentérologie et Hépatologie

1 place Ricordeau

Nantes 44000

France

Fax: +33240083154 emmanuel.coron@chu-nantes. $\mathrm{fr}$

\section{License terms}

Percutaneous endoscopic colostomy (PEC) was first described in 1986, by Jeffrey Ponski [1], who had invented percutaneous endoscopic gastrostomy a few years before that [2]. Performed by 2 operators, PEC consists of placing a tube in the colon during colonoscopy in order to provide antegrade colonic enemas (ACE) in severe constipation or fecal incontinence $[3,4]$. The PEC tube can also be used as a tool allowing easier and immediate colonic exsufflation in Ogilvie's syndrome or chronic intestinal obstruction $[5,6]$. In the majority of PEC indications, the colostomy tube is placed in the cecum for 2 main reasons: 1 ) to allow pancolonic rather than distal ACE in order to (theoretically) provide more effective bowel function; and 2) cecal transillumination as a prerequisite to improve procedure ease and safety because it helps determine the most direct route to the colon. So far, whether performing PEC is safe and effective in other situations, such as sigmoid volvulus, remains largely unknown.

What is the clinical problem with sigmoid volvulus and how can PEC fix it? First of all, sigmoid volvulus is not a rare disease because since it accounts for $7 \%$ of intestinal occlusions, being the third most common cause of colonic occlusion after cancer and diverticular disease [7]. Incidence of sigmoid volvulus is especially high in Africa, East Asia, and Eastern Europe, probably because of dietary habits in these countries. Besides high intake of fibers, other potential factors frequently associated with sigmoid volvulus are megacolon, age, male sex, and ethnicity. However, little is known regarding the pathophysiology of this disease, especially concerning the acute event precipitating the twist of the distal colon. In practice, the clinical symptoms often mimic small bowel obstruction but in most cases, plain abdominal radiograph is sufficient to diagnose the typical aspect of sigmoid volvulus. Emergency colonoscopy is a highly effective and safe option for untwisting the volvulus and exsufflating the proximal colon while avoiding ischemic necrosis and perforation. Indeed, the technical success of endoscopy is considered to be around $95 \%$ with a low morbidity and mortality $(<5 \%)$, even in aged and frail patients [8]. In contrast, immediate surgery is associated with high mortality rates and should be considered only in cases of septic shock, peritonitis or suspicion of severe colonic ischemia.

In fact, the biggest issue with sigmoid volvulus is the very high recurrence rate $(40 \%-90 \%)[8,9]$. The risk of recurrence can be prevented by prophylactic sigmoidectomy, but this strategy is generally proposed in a minority of cases because sigmoid volvulus is especially frequent in elderly and frail patients. Among the 83 patients with sigmoid volvulus whom we studied at Nantes University Hospital, $55 \%$ of those who refused surgery or in whom it was contraindicated went on to experience an average of 2.6 additional episodes of sigmoid volvulus over the next 18 months (personal data). Given that we will see more and more octogenarians and nonagenarians in the next decades, we should consider different strategies for preventing recurrence of sigmoid volvulus because the condition represents a burden not only for the endoscopist on call, but also for society in terms of hospital costs.

In this issue of Endoscopy International Open, Frank et al. [10] report on a systematic review of PEC performed in the sigmoid colon for the treatment of sigmoid volvulus in 56 patients. Unfortunately, it is difficult to estimate the efficacy of PEC in this situation because this review could only collect heterogeneous data from 5 case series and 5 observational studies, with only 1 study performed prospectively. The number of patients was low $(1-19)$ and the technique used for PEC was very different among studies. However, this review adds to our knowledge by providing novel and interesting insight regarding the feasibility and complications of PEC in this specific situation. 
Indeed, several important comments can be made. Firstly, PEC was feasible (although not easy to perform) in the sigmoid colon in most cases despite poor transillumination obtained at this level and frequently insufficient bowel cleansing. However, because PEC theoretically offers better visual control for placing fasteners and tubes than radiological procedures, it highlights the need for optimal preparation of patients and the colonoscopy environment. Secondly, introducer ("push") methods are safer than traction ("pull") methods because the latter are associated with higher risks of infections. Although no study directly compared these 2 approaches, several case reports or small series suggested that the rate of septic complications is higher with traction than with introducer methods, whether PEC is placed at the level of the cecum $[3,4]$ or more distally. Thirdly, striking differences exist regarding the number and type of tubes that were used for PEC in the review by Frank et al. For instance, the diameter ranged from 12 to $22 \mathrm{Fr}$, which is likely to play a role in the efficacy and tolerability of the implanted device. Although almost one-third of patients requested tube removal or had a tube removed, data are lacking to explain the exact reason for this. Also, it is interesting to note that 3 observational studies and 2 case reports used 2 PEC devices rather than 1. Although no definitive conclusion can be drawn with these small numbers of patients, it is tempting to speculate that placing PEC tubes at 2 different spots would ensure better fixation of the colon. However, the subtype of sigmoid volvulus, which was not investigated in these studies, might also play a role in the future choice of PEC spot. Indeed, from an anatomical point of view, 2 subtypes of sigmoid volvulus can be characterized using computerized tomography or (less and less frequently) by barium enema: 1) a twist of the sigmoid colon over its mesenteric axis, which is the most well-known subtype, resulting in the endoscopic aspect of "closed-loop" with 2 spiral stenosis; and 2) a twist of the sigmoid colon over its longitudinal axis, which was only discovered in 1992 but might account for up to $70 \%$ of occlusions [11] and results endoscopically in a single spiral stenosis. Whether these anatomical differences play a significant role regarding disease evolution and PEC efficacy remains unknown, as does the role of other parameters such as colonic dysmotility or enteric nerves alterations [12].

In conclusion, PEC is still far from standardization. Still unresolved questions include how to select the ideal spot for PEC, how many (and where) T-fasteners should be placed, which type of tube device to use, and how many tube devices to implant. Another important question is whether PEC should be used only as a fixing device or an access route to perform ACE on a regular basis in order to ensure bowel transit. All of these issues should be addressed by future prospective, and if possible multicenter studies to draw conclusions from large numbers of patients. Most importantly, investigating the potential efficacy and long-term benefit of PEC will require a large randomized controlled trial to compare PEC with no intervention, after a first episode of sigmoid volvulus. Better understanding of the etiology of sigmoid volvulus and pathophysiology is also necessary. Current- ly, PEC should not be recommended as a routine option but only proposed in selected cases following multidisciplinary assessment of inoperable patients. It should be performed by welltrained operators, if possible in tertiary referral centers. Because we will face a significant increase in the proportion of elderly patients, the issue of sigmoid volvulus should be addressed as soon as possible in order to determine whether it will become a standard therapeutic option in routine practice. Thirteen years after the invention of PEC, its story is just beginning.

Competing interests: Dr. Coron has served as a consultant for Cook Medical.

\section{Acknowledgements \\ $\nabla$}

The author thanks Drs. Marc Le Rhun and Nicolas Musquer as well as the endoscopy nurses at the Digestive Diseases Institute for their dedicated care of patients.

\section{References}

1 Ponsky JL, Aszodi A, Perse D. Percutaneous endoscopic cecostomy: a new approach to nonobstructive colonic dilation. Gastrointest Endosc 1986; 32: $108-111$

2 Gauderer MW, Ponsky JL, Izant RJ Jr. Gastrostomy without laparotomy: a percutaneous endoscopic technique. J Pediatr Surg 1980; 15: 872875

3 Uno $Y$. Introducer method of percutaneous endoscopic cecostomy and antegrade continence enema by use of the Chait Trapdoor cecostomy catheter in patients with adult neurogenic bowel. Gastrointest Endosc 2006; 63: 666-673

4 Duchalais E, Meurette G, Mantoo SK et al. Percutaneous endoscopic caecostomy for severe constipation in adults: feasibility, durability, functional and quality of life results at 1 year follow-up. Surg Endosc 2015; 29: $620-626$

5 Küllmer A, Schmidt A, Caca K. Percutaneous endoscopic cecostomy (introducer method) in chronic intestinal pseudo-obstruction: Report of two cases and literature review. Dig Endosc 2016; 28: 210-215

6 Lynch $C R$, Jones RG, Hilden $K$ et al. Percutaneous endoscopic cecostomy in adults: a case series. Gastrointest Endosc 2006; 64: 279-282

7 Weingrow D, McCague A, Ravi Shah $R$ et al. Acute volvulus of sigmoid colon. World J. Surg 1987; 11: 258-262

8 Oren D, Atamanalp SS, Aydinli B et al. An algorithm for the management of sigmoid volvulus and the safety of primary resection: experience with 827 cases. Dis. Colon Rectum 2007; 50: 489-497

9 Lal SK, Morgenstern R, Vinjirayer EP et al. Sigmoid volvulus an update. Gastrointest Endosc Clin N Am 2006; 16: 175-187

10 Frank L, Moran A, Beaton C. The use of percutaneous endoscopic colostomy to treat sigmoid volvulus. A systematic review. Endoscopy International Open 2016: DOI 10.1055/s-0042-106957 [Epub ahead of print]

11 Janzen $D L$, Stuart WH. Organo-axial volvulus of the sigmoid colon. Austral Radiology 1992; 36: $332-333$

12 Tomita R, Sugitou K, Sakurai K et al. Role of the enteric nervous system in the elongated sigmoid colon of patients with sigmoid volvulus. Int Surg 2014; 99: 699-704

13 Baraza W, Brown S, McAlindon $M$ et al. Percutaneous endoscopic sigmoidopexy: a cost-effective means of treating sigmoid volvulus in Sub-Saharan Africa? East Afr Med J 2007; 84: 1 -2 